\title{
MIXING PROPERTIES FOR STIT TESSELLATIONS
}

\author{
R. LACHIÈZE-REY, ${ }^{*}$ Université des Sciences et Technologies de Lille
}

\begin{abstract}
The so-called STIT tessellations form a class of homogeneous (spatially stationary) tessellations in $\mathbb{R}^{d}$ which are stable under the nesting/iteration operation. In this paper we establish the mixing property for these tessellations and give the decay rate of $\mathrm{P}\left(A \cap M=\varnothing, T_{h} B \cap M=\varnothing\right) / \mathrm{P}(A \cap Y=\varnothing) \mathrm{P}(B \cap Y=\varnothing)-1$, where $A$ and $B$ are both compact connected sets, $h$ is a vector of $\mathbb{R}^{d}, T_{h}$ is the corresponding translation operator, and $M$ is a STIT tessellation.
\end{abstract}

Keywords: Stochastic geometry; random tessellation; STIT tessellation; space ergodicity; mixing property

2010 Mathematics Subject Classification: Primary 60D05

Secondary 05B $45 ; 37 \mathrm{~A} 25$

\section{Introduction and notation}

Random tessellations, or mosaics, form an important class of objects from stochastic geometry. They have proven to be a useful tool for modelling geometrical structures appearing in biology, geology, and medical sciences. The Poisson hyperplane tessellation, the PoissonVoronoi tessellation, and its dual, the Poisson-Delaunay tessellation, are the most celebrated and tractable models investigated to date, and all are defined using a Poisson point process on an appropriate space. In the 1980s, Ambartzumian had the idea of applying an operation to the mosaics of $\mathbb{R}^{d}$, namely the operation of iteration (also called the nesting operation). In $\mathbb{R}^{2}$, the class of T-noded tessellations is stable under iteration, while the class of X-noded tessellations, such as the Poisson line tessellation, is stable under superposition. Nagel, Mecke, and Weiss (see [6], [7], and [8]) introduced the STIT (stable under iteration) tessellation model, motivated by Cowan's [3] work. The STIT tessellation can be used as a model for crack patterns, such as those seen on old pottery or on drying soil.

The STIT tessellation is homogeneous, i.e. space stationary, and a proper choice of parameters can make it isotropic, which yields a very interesting model. Many geometrical features of STIT tessellations have been investigated, including moments of variables related to typical faces of the tessellation. Cowan [1], [2] emphasised the importance of ergodic properties for random tessellations. In this paper we establish that all STIT tessellations possess the mixing property, which implies ergodicity. Namely, if $A$ and $B$ are two Borel sets, and $M$ is the closed set of boundaries of the cells of a STIT tessellation, then

$$
\mathrm{P}\left(M \cap A=\varnothing, M \cap T_{h} B=\varnothing\right)-\mathrm{P}(M \cap A=\varnothing) \mathrm{P}(M \cap B=\varnothing) \rightarrow 0
$$

as $\|h\| \rightarrow+\infty$, where $T_{h} B$ is the set $B$ translated by the vector $h$. In the general case, the decay is $o\left(\|h\|^{-1}\right)$.

Received 18 May 2009; revision received 24 September 2010.

* Postal address: Laboratoire de Statistique et Probabilités, UFR de Mathematiques Bat. M2, Université des Sciences et Technologies de Lille, 59655 Villeneuve d'Ascq, France. Email address: 1r.raphael@ gmail.com 
The paper is organised as follows. In the rest of this section we introduce the notation we will use in the following sections. In Section 2 we give a brief description of the construction and the properties of STIT tessellations. In Section 3 we establish mixing properties for STIT tessellations. Section 4 contains the proofs of the results obtained in the paper.

\subsection{Notation}

In the sequel, $\operatorname{int}(A)$ denotes the interior of a set $A, \operatorname{conv}(A)$ denotes its convex hull, $\partial A$ denotes its boundary, and $\operatorname{span}(A)$ denotes the smallest subspace of $\mathbb{R}^{d}$ containing $A$.

Since we are interested in hitting and missing probabilities here, we introduce the corresponding families. For $A \in \mathscr{B}\left(\mathbb{R}^{d}\right)$,

$$
\mathcal{F}_{A}=\{C \in \mathcal{F}: C \cap A \neq \varnothing\}, \quad \mathcal{F}^{A}=\{C \in \mathcal{F}: C \cap A=\varnothing\} .
$$

Denote by $\mathcal{K}$ the class of compact sets of $\mathbb{R}^{d}$. The topology of closed convergence on $\mathcal{F}$, or the Fell topology, is the topology generated by $\mathcal{F}^{K}, K \in \mathcal{K}$, and $\mathcal{F}_{O}$, for open $O$. For any subclass $\mathcal{C} \subset \mathcal{F}$, we can define the induced Fell topology and the corresponding Borel $\sigma$-algebra $\mathscr{B}(\mathcal{C})$. It is known that the $\sigma$-algebra $\mathscr{B}(\mathcal{F})$ is generated by the $\mathcal{F}^{K}, K \in \mathcal{K}$; see [9, Lemma 2.1.1]. Let us give definitions for tessellations and random tessellations.

A tessellation of $\mathbb{R}^{d}$ is a countable set $R$ of convex polytopes of $\mathbb{R}^{d}$ that satisfies

(i) for all $C \in R, \operatorname{int}(C) \neq \varnothing$;

(ii) $\bigcup_{C \in R} C=\mathbb{R}^{d}$;

(iii) for all $C, C^{\prime} \in R$ such that $C \neq C^{\prime}$, we have $\operatorname{int}(C) \cap \operatorname{int}\left(C^{\prime}\right)=\varnothing$;

(iv) for all $K \in \mathcal{K}, \operatorname{card}\left(\mathcal{F}_{K} \cap R\right)<\infty$.

It is equivalent and more convenient to work with the closed set $M=\bigcup_{C \in R} \partial C$ formed by the boundaries of the cells of $R$. Reciprocally, it is possible to retrieve $R$ from $M$ by taking the closures of all connected components of $M$ 's complement. Call $\mathcal{M}$ the subclass of $\mathcal{F}$ formed by all tessellations.

A random tessellation is a random element with values in $(\mathcal{M}, \mathcal{B}(\mathcal{M}))$. According to the Choquet theorem (see [5] and [9, Theorem 2.2.1]), the law $\mathrm{P}$ of a random closed set $M$ is characterised by its capacity functional, defined by

$$
\mathfrak{T}_{M}(K)=\mathrm{P}\left(\mathcal{F}_{K}\right), \quad K \in \mathcal{K} .
$$

The equality in law, denoted by ' $\stackrel{\mathrm{D}}{=}$, occurs if and only if there is equality of the corresponding capacity functionals.

\section{STIT tessellations}

A STIT tessellation, as a Poisson hyperplane tessellation, is constructed from a hyperplane Poisson process. Details concerning the construction of STIT tessellations can be found in [6], [7], and [8].

We give here the construction of a STIT tessellation seen through a compact window $W$. We do not use the elegant construction from a hyperplane Poisson process, because it is less explicit, but the reader is referred to [7] for such a description. Let us begin with some definitions.

Call $\mathscr{H}$ the class of all hyperplanes of $\mathbb{R}^{d}$. For $A \in \mathscr{B}$, set

$$
[A]=\mathscr{H} \cap \mathcal{F}_{A},
$$


the family of all hyperplanes hitting $A$. For another Borel set $B$, define $[A \mid B]$ as the family of all hyperplanes $\gamma$ that strictly separate $A$ and $B$, i.e. such that $A$ and $B$ are contained in different open half-spaces of $\mathbb{R}^{d} \backslash \gamma$. We write in short, for $x, y \in \mathbb{R}^{d}$,

$$
[x]=[\{x\}], \quad[x \mid y]=[\{x\} \mid\{y\}] .
$$

Given $\gamma \in \mathscr{H} \backslash[0]$, denote by $\gamma^{+}$the closed half-space delimited by $\gamma$ not containing 0 , and denote by $\gamma^{-}$the other closed half-space. Let $\delta^{d-1}$ be the unit sphere of $\mathbb{R}^{d}$. We identify $\mathscr{H}$ with $\mathbb{R}^{+} \times \delta^{d-1}$, where an element $(r, u)$ of $\mathbb{R}^{+} \times \delta^{d-1}$ stands for the hyperplane $\gamma$ at distance $r$ from the origin with normal exterior vector $u$ (i.e. a normal vector directed towards $\gamma^{+}$). If $\gamma \in[0]$, we take the arbitrary convention that $\gamma^{+}$is the half-space that contains $(1,0, \ldots, 0)$ in its interior, or $(0, \ldots, 0,1)$ if $(1,0, \ldots, 0) \in \gamma$.

Equip $\mathscr{H}$ with the topology inherited from this identification and the corresponding $\sigma$-algebra. Let $\Lambda$ be a translation invariant locally finite measure on $\mathscr{H}$. Note that $\Lambda([0])=0$, since $\Lambda$ is stationary and locally finite. By stationarity we can write, along with the identification,

$$
\Lambda=\lambda^{+} \otimes \nu
$$

where $\lambda^{+}$is the Lebesgue measure restricted to $\mathbb{R}^{+}$and $v$ is a finite measure on $\delta^{d-1}$. Also, assume that

$$
\operatorname{span}(\operatorname{supp}(v))=\mathbb{R}^{d} .
$$

The latter property ensures that tessellations generated by the hyperplane processes with distribution $\Lambda$ will almost surely have compact cells (whether it is a STIT tessellation or a Poisson hyperplane tessellation).

Let $a$ be a positive number. On a compact window $W \in \mathcal{K}$ with nonempty interior, we define the tessellation with parameters $a$ and $\Lambda$, where $a$ is the time parameter, as a stochastic process taking values in $\mathcal{M}$. Let $\mathbb{B}$ be Young's binary infinite tree, where the leaves are labelled so that the top leaf is attributed the number 1 , and the $k$-labelled leaf has $2 k$ - and $(2 k+1)$ labelled daughter leaves. We now attach to each leaf a pair $\left(\epsilon_{k}, \gamma_{k}\right)$, where $\left(\epsilon_{k}\right)_{k \geq 1}$ is a family of independent and identically distributed (i.i.d.) random exponential variables with parameter $\Lambda([W])$, and the $\left(\gamma_{k}\right)_{k \geq 1}$ are i.i.d. random hyperplanes with law $\Lambda([W] \cap \cdot) / \Lambda[W]$.

The tessellation is defined as a process of cell division, where each cell is identified with a leaf of the tree, and the first cell is the window $C_{1}=W$ itself. We describe the tesselation in terms of a birth-and-death process, where a cell dies when it is divided into two daughter cells. The death times $\left(d_{k}\right)$, birth times $\left(b_{k}\right)$, and daughter cells of $\left(C_{k}\right)_{k \geq 1}$ are defined recursively as follows (here $\lfloor s\rfloor$ is the integer part of $s$ ):

$$
b_{1}=0, \quad b_{k}=d_{\lfloor k / 2\rfloor}, \quad d_{k}=b_{k}+\epsilon_{k}, \quad C_{2 k}=C_{k} \cap \gamma_{k}^{-}, \quad C_{2 k+1}=C_{k} \cap \gamma_{k}^{+} \text {. }
$$

We then define

$$
M_{a, W}=\bigcup_{b_{k} \leq a} \partial C_{k} .
$$

In the sequel we denote the law of this tessellation by $\mathrm{P}_{a, W}$. Note that $\Lambda$ is an implicit parameter of the model. We also write for short

$$
\mathfrak{T}_{a, W}=\mathfrak{T}_{M_{a, W}} \quad \text { and } \quad \mathfrak{U}_{a, W}=1-\mathfrak{T}_{a, W} .
$$


Remarks. 1 . The only nonintuitive feature of this construction is that $\gamma_{k}$ might not be in $\left[C_{k}\right]$, which would mean that one daughter cell is $\varnothing$ and the other is $C_{k}$ itself. There is no theoretical objection to this feature, but it can be remedied in the following way. Instead of choosing an i.i.d. family $\left(\epsilon_{k}\right)$, independently attach to each cell $C_{k}$ the death rate $\Lambda\left(\left[C_{k}\right]\right)$ and a hyperplane drawn from $\Lambda\left(\left[C_{k}\right] \cap \cdot\right) / \Lambda\left[C_{k}\right]$. The resulting law of the tessellation is not modified and each hyperplane indeed hits the cell to which it is attached.

2. If $\Lambda$ is isotropic, the death rate $\Lambda([C])$ of a cell $C$ is proportional to the perimeter of $C$.

3. We now mention a construction from a Poisson hyperplane process. Let $\Theta$ be a Poisson process on $\mathbb{R}^{+} \times \mathscr{H}$ with intensity $\Lambda([W]) \lambda^{+} \otimes \Lambda([W] \cap \cdot) / \Lambda[W]$. Since, almost surely, for all $t \geq 0, \operatorname{card}((\{t\} \times \mathscr{H}) \cap \Theta) \leq 1$, we can define a random sequence $\left(\tau_{k}, \gamma_{k}\right)_{k \geq 1}$ such that $\tau_{k}<\tau_{k+1}$ for all $k \geq 1, \Theta=\left\{\left(\tau_{k}, \gamma_{k}\right) \mid k \geq 1\right\}$, and $\left(\tau_{k+1}-\tau_{k}, \gamma_{k}\right)_{k \geq 1}$ satisfy the hypotheses of the previous construction almost surely.

Nagel and Weiss [8] established the consistency property.

Theorem 1. If $W \subset W^{\prime}$ are two compact sets with nonempty interiors then

$$
M_{a, W} \cap \operatorname{int}(W) \stackrel{\mathrm{D}}{=} M_{a, W^{\prime}} \cap \operatorname{int}(W) .
$$

The statement of Theorem 1 means that, for any compact set $K \subset \operatorname{int}(W), \mathfrak{T}_{a, W}(K)=$ $\mathfrak{T}_{a, W^{\prime}}(K)$, and on $\mathcal{K}$ we can define

$$
\mathfrak{T}_{a}(K)=\mathfrak{T}_{a, W}(K), \quad \mathfrak{U}_{a}(K)=\mathfrak{U}_{a, W}(K), \quad \text { for any } W \text { containing } K \text { in its interior. }
$$

Theorem 2.3.1 of [9], which is a consequence of the Choquet theorem, allows us to define a tessellation $M_{a}$ on the whole space, with capacity functional $\mathfrak{T}_{a}$, such that

$$
M_{a} \cap \operatorname{int}(W) \stackrel{\mathrm{D}}{=} M_{a, W} \cap \operatorname{int}(W), \quad W \in \mathcal{K},
$$

which is called the STIT tessellation with parameters $a$ and $\Lambda$. Denote by $\mathrm{P}_{a}$ its law on $\mathcal{M}$. Mecke et al. [6] also provided an explicit global construction on $\mathbb{R}^{d}$, i.e. without the help of a general extension theorem.

\section{Mixing property}

Here $\Lambda=\lambda^{+} \otimes v$ is a measure on $\mathscr{H}$ satisfying assumption (2) and $a$ is a strictly positive number.

Consider the set $\mathcal{T}=\left\{T_{h} ; h \in \mathbb{R}^{d}\right\}$ of all translations, seen as operators on $\mathcal{F}$. Their action is naturally lifted to $\mathcal{B}(\mathcal{F})$, for which we keep the same notation. The stationarity of a random closed set $X$ with law $\mathrm{P}$ means that, for all $T \in \mathcal{T}$, P is invariant under $T$. Now we call every Borel set $\mathcal{C}$ of the $\sigma$-algebra $\mathscr{B}(\mathcal{F})$ a $\mathcal{T}$-invariant set of $\mathcal{B}(\mathcal{F})$ if, for all $T$ in $\mathcal{T}, T \mathcal{C}=\mathcal{C}$. For instance, $\mathcal{K}, \mathscr{H}, \mathcal{M}$, or 'the class of all tessellations having a cube as one of their cells' are invariant sets. Given a stationary law $\mathrm{P}$ on $\mathcal{F}$, the dynamical system $(\mathcal{F}, \mathcal{B}(\mathcal{F}), \mathrm{P}, \mathcal{T})$ is said to be ergodic if every $\mathcal{T}$-invariant set has probability 0 or 1 , and mixing if

$$
\mathrm{P}\left(\mathcal{C} \cap T_{h} \mathcal{C}^{\prime}\right) \rightarrow \mathrm{P}(\mathcal{C}) \mathrm{P}\left(\mathcal{C}^{\prime}\right) \quad \text { as }\|h\| \rightarrow+\infty
$$

for all $\mathcal{C}, \mathcal{C}^{\prime}$ in $\mathcal{F}$. If $X$ is a random closed set with law $\mathrm{P}$, we simply speak of the stationarity, the ergodicity, or the mixing property for $X$. Roughly speaking, the mixing property yields 
the asymptotic independence of relatively distant sets. Since any $\mathcal{T}$-invariant set $\mathcal{C}$ satisfies $\mathrm{P}\left(\mathcal{C} \cap T_{h} \mathcal{C}\right)=\mathrm{P}(\mathcal{C})$, the mixing property implies ergodicity.

According to Lemma 9.3.1 of [9], it suffices to show (3) for sets $\mathcal{C}, \mathcal{C}^{\prime}$ drawn from a semialgebra $\mathcal{A}$ generating $\mathcal{B}(\mathcal{F})$. Therefore, the mixing property for $M_{a}$ is a consequence of the following theorem.

Theorem 2. For all $A, B \in \mathscr{B}\left(\mathbb{R}^{d}\right)$,

$$
\mathrm{P}_{a}\left(\mathcal{F}^{A}, \mathcal{F}^{T_{h} B}\right) \rightarrow \mathrm{P}_{a}\left(\mathcal{F}^{A}\right) \mathrm{P}_{a}\left(\mathcal{F}^{B}\right) \quad \text { as }\|h\| \rightarrow \infty .
$$

As $T_{h} \mathcal{F}^{B}=\mathcal{F}^{T^{-h} B}$, we state the theorem with $h$ instead of $-h$ for more simplicity, since the role of $h$ is symmetric. Since the $\left\{\mathcal{F}^{K} ; K \in \mathcal{K}\right\}$ also generate the Borel $\sigma$-algebra of the Fell topology, with the help of Theorem 9.3.2 of [9], which is a consequence of [9, Lemma 9.3.1], it suffices to show Theorem 2 for $A, B \in \mathcal{K}$. (It was first proved in [4].) Thus, this theorem is a consequence of Theorem 3 below.

Theorem 3. For all $A, B \in \mathcal{K}$,

$$
\left|\mathrm{P}_{a}\left(\mathcal{F}^{A}, \mathcal{F}^{T_{h} B}\right)-\mathrm{P}_{a}\left(\mathcal{F}^{A}\right) \mathrm{P}_{a}\left(\mathcal{F}^{B}\right)\right|=O\left(\|h\|^{-1}\right) .
$$

To give this bound, we have to estimate the Lipschitz constant for the capacity functional as a function of $a$. We obtain the following result.

Proposition 1. For all compact sets $K$, there exists $a \beta_{K, a}>0$ such that

$$
0 \leq \mathfrak{T}_{a+t}(K)-\mathfrak{T}_{a}(K) \leq t \beta_{K, a}, \quad t>0 .
$$

Furthermore, $\beta_{K, a}$ is invariant under rigid motions of $K$.

\section{Proofs}

\subsection{Proof of Proposition 1}

Lemma 1. Let $0<t$, a be real positive numbers, let $K$ be a compact set, and let

$$
\beta_{K, a}=\Lambda([\operatorname{conv}(K)])(1+a \Lambda([\operatorname{conv}(K)]))\left(1-\mathfrak{T}_{a}(K)\right) .
$$

Then $0 \leq \mathfrak{T}_{a+t}(K)-\mathfrak{T}_{a}(K) \leq t \beta_{K, a}$.

Proof. We use the notation of Section 2, with $W=\operatorname{conv}(K)$. Let $N_{a}=\operatorname{card}\left\{k ; \varepsilon_{k} \leq a\right\}$ denote the number of times conv $(K)$ has been 'hit' by a hyperplane up to time $a$ (if the hyperplane falls outside $\operatorname{conv}(K)$, it is still counted as a hit). For $n \in \mathbb{N}$, set $\pi_{n}(a)=\mathrm{P}\left(N_{a}=n\right)$. Almost surely, conditionally on $\left(N_{a}=n\right), M_{a, W}$ has $n+1$ cells $C_{1}, \ldots, C_{n+1}$, not necessarily distinct, with nonempty interiors. Define $K_{i}=K \cap C_{i}$. Then

$$
\begin{aligned}
\mathfrak{T}_{a+t}(K)-\mathfrak{T}_{a}(K) \\
=\mathfrak{U}_{a}(K)-\mathfrak{U}_{a+t}(K) \\
=\mathfrak{U}_{a}(K) \mathrm{P}\left(K \cap M_{a+t} \neq \varnothing \mid K \cap M_{a}=\varnothing\right) \\
=\mathfrak{U}_{a}(K) \\
\quad \times \sum_{n \geq 0} \pi_{n}(a) \mathrm{P}\left(\text { there exists } i, 1 \leq i \leq n+1, K_{i} \cap M_{a+t} \neq \varnothing \mid N_{a}=n, K \cap M_{a}=\varnothing\right) \\
\leq \mathfrak{U}_{a}(K) \sum_{n \geq 0} \pi_{n}(a) \sum_{i=1}^{n+1} \mathrm{P}\left(K_{i} \cap M_{a+t} \neq \varnothing \mid N_{a}=n, K \cap M_{a}=\varnothing\right) .
\end{aligned}
$$


Let us now recall some essential facts concerning the construction of a STIT tessellation. When a cell is divided, the two daughter cells behave independently. Moreover, the division process of a cell has no memory, which means that the probability that it is divided at time $a+t$ knowing it has not been divided at time $a$ equals the probability that it is divided within a period of length $t$. Since, for each $i, K_{i}$ is contained in the convex cell $C_{i}$, the probability that $K_{i}$ is divided within a certain period equals the probability that the STIT tessellation defined on $C_{i}$ touches $K_{i}$ within a period with the same length. Finally, owing to the consistency property, conditionally on $C_{i}=C_{i}^{0}$, for some $C_{i}^{0}$ in $\mathcal{K}$, the latter probability does not change if the tessellation is considered a random object of $\mathbb{R}^{d}$ (meaning that the value of $C_{i}^{0}$ does not matter). Thus, we have, for every compact $K$,

$$
\begin{aligned}
\mathrm{P}\left(\operatorname{conv}\left(K_{i}\right) \cap M_{a+t} \neq \varnothing \mid N_{a}=n, \operatorname{conv}\left(K_{i}\right) \cap M_{a}=\varnothing\right) & =1-\mathfrak{U}_{t}\left(\operatorname{conv}\left(K_{i}\right)\right) \\
& \leq 1-\mathfrak{U}_{t}(\operatorname{conv}(K)) \\
& \leq 1-\mathrm{e}^{-t \Lambda([\operatorname{conv}(K)])} .
\end{aligned}
$$

This yields

$$
\begin{aligned}
\mathfrak{U}_{a}(K)-\mathfrak{U}_{a+t}(K) & \leq \mathfrak{U}_{a}(K) \sum_{n \geq 0} \pi_{n}(a) \sum_{i=1}^{n+1}\left(1-\mathrm{e}^{-t \Lambda([\operatorname{conv}(K)])}\right) \\
& \leq \mathfrak{U}_{a}(K) \sum_{n \geq 0} \pi_{n}(a) t \sum_{i=1}^{n+1} \Lambda([\operatorname{conv}(K)]) \\
& \leq \mathfrak{U}_{a}(K) \sum_{n \geq 0} \pi_{n}(a)(n+1) t \Lambda([\operatorname{conv}(K)]) \\
& \leq \mathfrak{U}_{a}(K) t \Lambda([\operatorname{conv}(K)])\left(1+\mathrm{E}\left(N_{a}\right)\right) .
\end{aligned}
$$

The number of hyperplanes involved in the cell division process with initial cell $\operatorname{conv}(K)$ up to time $a$ is a Poisson variable with parameter $a \Lambda([\operatorname{conv}(K)])$. Therefore,

$$
\mathrm{E}\left(N_{a}\right)=a \Lambda([\operatorname{conv}(K)])
$$

which concludes the proof.

\subsection{Proof of Theorem 3}

We first establish some inequalities for compact sets $A$ and $B$, and then add a drift $h$ to give an upper bound when the expressions become too complicated.

Let $W=\operatorname{conv}(A \cup B)$. The key is to consider the tessellation-valued time process $\left(M_{t, W}\right)_{t \geq 0}$ defined in Section 2. We have the almost-sure identity

$$
\epsilon_{1}=\inf \left\{t: M_{0, W} \neq M_{t, W}\right\} .
$$

Let $\gamma_{1}$ be the first hyperplane dividing $C_{1}=W$. We introduce the event

$$
\Gamma_{A, B}=\left\{\gamma_{1} \in[A \mid B], \epsilon_{1} \leq a\right\} .
$$

If $A$ and $B$ are far away from each other, $\Gamma_{A, B}$ is likely to happen, and after $\Gamma_{A, B}$ occurs, the tessellations inside $A$ and $B$ behave independently because they are in disjoint cells. Then

$$
\begin{aligned}
\left|\mathrm{P}_{a}\left(\mathcal{F}^{A}, \mathcal{F}^{B}, \Gamma_{A, B}\right)-\mathrm{P}_{a}\left(\mathcal{F}^{A}, \mathcal{F}^{B}\right)\right| & \leq \mathrm{P}\left(\gamma_{1} \notin[A \mid B]\right)+\mathrm{P}_{a}\left(\varepsilon_{1}>a\right) \\
& \leq \frac{\Lambda([A])+\Lambda([B])}{\Lambda([W])}+\mathrm{e}^{-a \Lambda([W])}
\end{aligned}
$$


Consequently, we can show (4) with $\mathrm{P}_{a}\left(\mathcal{F}^{A}, \mathcal{F}^{T_{h} B}, \Gamma_{A, T_{h} B}\right)$ instead of $\mathrm{P}_{a}\left(\mathcal{F}^{A}, \mathcal{F}^{T_{h} B}\right)$, because their difference has the same magnitude than the expected decay rate. Rigorously, this gives

$$
\begin{aligned}
\mathrm{P}_{a}\left(\mathcal{F}^{A}, \mathcal{F}^{B}, \Gamma_{A, B}\right) & =\int_{0}^{a} \mathrm{P}_{a}\left(\epsilon_{1} \in \mathrm{d} t, \gamma_{1} \in[A \mid B], \mathcal{F}^{A}, \mathcal{F}^{B}\right) \\
& =\int_{0}^{a} \mathrm{P}_{a}\left(\epsilon_{1} \in \mathrm{d} t, \gamma_{1} \in[A \mid B]\right) \mathrm{P}_{a}\left(\mathcal{F}^{A}, \mathcal{F}^{B} \mid \epsilon_{1}=t, \gamma_{1} \in[A \mid B]\right) .
\end{aligned}
$$

If $\epsilon_{1}=t$ and $\gamma_{1} \in[A \mid B]$, then $A$ and $B$ are not hit up to time $t$, and are both still contained in a cell (but the cell encapsulating $A$ is different from that encapsulating $B$ ). Owing to the consistency property, and the independent behaviour of distinct cells after their birth, we have

$$
\mathrm{P}_{a}\left(\mathcal{F}^{A}, \mathcal{F}^{B} \mid \epsilon_{1}=t, \gamma_{1} \in[A \mid B]\right)=\mathfrak{U}_{a-t}(A) \mathfrak{U}_{a-t}(B) .
$$

Since the sequence $\left(\gamma_{k}\right)_{k \geq 1}$ is independent of $\left(\epsilon_{k}\right)_{k \geq 1}$, we have

$$
\begin{aligned}
\mathrm{P}_{a}\left(\epsilon_{1} \in \mathrm{d} t, \gamma_{1} \in[A \mid B]\right) & =\mathrm{P}_{a}\left(\epsilon_{1} \in \mathrm{d} t\right) \mathrm{P}_{a}\left(\gamma_{1} \in[A \mid B]\right) \\
& =\Lambda([W]) \mathrm{e}^{-t \Lambda([W])} \mathrm{d} t \frac{\Lambda([A \mid B])}{\Lambda([W])},
\end{aligned}
$$

and, finally, we have

$$
\mathrm{P}_{a}\left(\mathcal{F}^{A}, \mathcal{F}^{B}, \Gamma_{A, B}\right)=\Lambda([A \mid B]) \int_{0}^{a} \mathrm{e}^{-t \Lambda([W])} \mathfrak{U}_{a-t}(A) \mathfrak{U}_{a-t}(B) \mathrm{d} t .
$$

We give the following relation, which will be useful in subsequent estimations:

$$
\Lambda([W])=\Lambda([A \mid B])+\Lambda([A])+\Lambda([B])-\Lambda([A] \cap[B]) .
$$

In order to make a proper upper bound estimate, consider a translation of $B$. In what follows, we use $T_{h} B$ instead of $B$ and let $W_{h}=\operatorname{conv}(\cdot)$. For $u \in \varsigma^{d}$, define $\zeta(u)=\Lambda([0, u])=$ $\Lambda([0 \mid u])$ and let

$$
\xi(h)=\|h\| \zeta\left(\frac{h}{\|h\|}\right), \quad h \in \mathbb{R}^{d} \backslash\{0\} .
$$

It is a standard fact from integral geometry that $\zeta$ is continuous, and it does not vanish because of assumption (2).

Lemma 2. We have

$$
\Lambda\left(\left[W_{h}\right]\right)=\xi(h)+o(\|h\|) \text { and } \Lambda\left(\left[A \mid T_{h} B\right]\right)=\xi(h)+o(\|h\|) .
$$

Proof. Let $\alpha \in A$ and $\beta \in B$. A hyperplane hitting $W_{h}$ that does not separate $\alpha$ and $\beta+h$ either hits $\operatorname{conv}(A)$ or $\operatorname{conv}\left(T_{h} B\right)$. Hence,

$$
\begin{aligned}
\left|\Lambda\left(\left[W_{h}\right]\right)-\Lambda([\alpha, \beta+h])\right| & \leq \Lambda([\operatorname{conv}(A)])+\Lambda\left(\left[\operatorname{conv}\left(T_{h} B\right)\right]\right) \\
& \leq \Lambda([\operatorname{conv}(A)])+\Lambda([\operatorname{conv}(B)]) \\
& =o(\|h\|) .
\end{aligned}
$$

Similarly,

$$
\left|\Lambda\left(\left[A \mid T_{h} B\right]\right)-\Lambda([\alpha, \beta+h])\right|=o(\|h\|)
$$


We only need to prove that

$$
\Lambda([\alpha, \beta+h])=\zeta\left(\frac{h}{\|h\|}\right)\|h\|(1+o(1))
$$

It suffices to show that if $\beta-\alpha=\|\beta-\alpha\| u$, with $u \in 8^{d-1}$,

$$
\Lambda([\alpha \mid \beta])=\|\beta-\alpha\| \zeta(u)
$$

This would follow from

$$
\Lambda([0 \mid(n+1) \epsilon u])=\Lambda([0 \mid n \epsilon u])+\Lambda([0 \mid \epsilon u])
$$

for all $\epsilon>0, u \in \varsigma^{d-1}$, and $n \in \mathbb{N}$. Indeed, since $\Lambda$ is stationary and locally finite, for all $x \in$ $\mathbb{R}^{d}, \Lambda([x])=0$, and then we will be able to obtain the result by induction and approximation. Since $[0 \mid(n+1) \epsilon u]$ is the disjoint union of $[0 \mid n \epsilon u],[n \epsilon u \mid(n+1) \epsilon u]$, and $[n \epsilon u]$, we have

$$
\Lambda([0 \mid(n+1) \epsilon u])=\Lambda([0 \mid n \epsilon u])+\Lambda([n \epsilon u \mid(n+1) \epsilon u]),
$$

and the result follows by stationarity of $\Lambda$.

The continuity of $\zeta$ yields

$$
\Lambda\left(\left[W_{h}\right]\right)=\|\alpha-\beta+h\| \zeta\left(\frac{\alpha-\beta+h}{\|\alpha-\beta+h\|}\right)(1+o(1))=\|h\| \zeta\left(\frac{h}{\|h\|}\right)(1+o(1)) .
$$

Thus, $\Lambda\left(\left[W_{h}\right]\right)=O\left(\|h\|^{-1}\right)$, and (7) gives

$$
\begin{aligned}
\mid \mathrm{P}_{a}\left(\mathcal{F}^{A},\right. & \left.\mathcal{F}^{T_{h} B}, \Gamma_{A, T_{h} B}\right)-\mathrm{P}_{a}\left(\mathcal{F}^{A}\right) \mathrm{P}_{a}\left(\mathcal{F}^{T_{h} B}\right) \mid \\
\leq & \Lambda\left(\left[A \mid T_{h} B\right]\right) \int_{0}^{\infty} \mathrm{e}^{-t \Lambda\left(\left[W_{h}\right]\right)}\left|\mathfrak{U}_{a-t}(A) \mathfrak{U}_{a-t}\left(T_{h} B\right)-\mathfrak{U}_{a}(A) \mathfrak{U}_{a}\left(T_{h} B\right)\right| \mathrm{d} t \\
& +\left|\frac{\Lambda\left(\left[A \mid T_{h} B\right]\right)}{\Lambda\left(\left[W_{h}\right]\right)}-1\right| \int_{0}^{\infty} \mathrm{e}^{-t \Lambda\left(\left[W_{h}\right]\right)} \mathfrak{U}_{a}(A) \mathfrak{U}_{a}\left(T_{h} B\right) \Lambda\left(\left[W_{h}\right]\right) \mathrm{d} t \\
& +O\left(\Lambda\left(\left[W_{h}\right]\right)^{-1} \mathrm{e}^{-a \Lambda\left(\left[W_{h}\right]\right)}\right) .
\end{aligned}
$$

Using (5) and (8), (9), and Lemma 2, we obtain

$$
\begin{aligned}
\left|\mathrm{P}_{a}\left(\mathcal{F}^{A}, \mathcal{F}^{T_{h} B}, \Gamma_{A, T_{h} B}\right)-\mathrm{P}_{a}\left(\mathcal{F}^{A}\right) \mathrm{P}_{a}\left(\mathcal{F}^{B}\right)\right| \\
\quad \leq \Lambda\left(\left[A \mid T_{h} B\right]\right)\left(\lambda_{A, a}+\lambda_{B, a}\right) \int_{0}^{\infty} \mathrm{e}^{-t \Lambda\left(\left[W_{h}\right]\right)} t \mathrm{~d} t+\left(1-\frac{\Lambda\left(\left[A \mid T_{h} B\right]\right)}{\Lambda\left(\left[W_{h}\right]\right)}\right)+o\left(\|h\|^{-1}\right) \\
\quad \leq \frac{\left(\lambda_{A, a}+\lambda_{B, a}\right) \Lambda\left(\left[A \mid T_{h} B\right]\right)}{\Lambda\left(\left[W_{h}\right]\right)^{2}}+\frac{\Lambda([\operatorname{conv}(A)])+\Lambda\left(\left[\operatorname{conv}\left(T_{h} B\right)\right]\right)}{\Lambda\left(\left[W_{h}\right]\right)}+o\left(\|h\|^{-1}\right) \\
\quad=O\left(\|h\|^{-1}\right) .
\end{aligned}
$$

Thus, (4) is proved.

\section{Acknowledgements}

I am grateful to Werner Nagel for useful discussion and comments. I also thank the anonymous referees for their constructive remarks and comments. Youri Davydov, my $\mathrm{PhD}$ advisor, and Ilya Molchanov helped to improve the paper with careful readings and remarks. 


\section{References}

[1] Cowan, R. (1978). The use of the ergodic theorems in random geometry. Suppl. Adv. Appl. Prob. 10, $47-57$.

[2] Cowan, R. (1980). Properties of ergodic random mosaic processes. Math. Nachr. 97, 89-102.

[3] Cowan, R. (1984). A collection of problems in random geometry. In Stochastic Geometry, Geometric Statistics, Stereology, eds R. V. Ambartzuminan and W. Weil, Teubner, Leipzig, pp. 64-68.

[4] Heinrich, L. (1992). On existence and mixing properties of germ-grain models. Statistics 23, 271-286.

[5] Matheron, G. (1975). Random Sets and Integral Geometry. John Wiley, New York.

[6] Mecke, J., NAGEL, W. AND Weiss, V. (2008). A global construction of homogeneous random planar tessellations that are stable under iteration. Stochastics 80, 51-67.

[7] Mecke, J., Nagel, W. And Weiss, V. (2008). The iteration of random tessellations and a construction of a homogeneous process of cell divisions. Adv. Appl. Prob. 40, 49-59.

[8] NAGEL, W. AND WEISs, V. (2005). Crack STIT tessellations: characterization of stationary random tessellations stable with respect to iteration. Adv. Appl. Prob. 37, 859-883.

[9] SchneIder, R. AND WeIL, W. (2008). Stochastic and Integral Geometry. Springer, Berlin. 